\title{
E-Learning for Improving Preterm Care
}

\author{
Adhisivam Bethou' ${ }^{1}$
}

Received: 10 September 2021 / Accepted: 14 September 2021 / Published online: 24 September 2021

(c) Dr. K C Chaudhuri Foundation 2021

Of the 15 million babies born preterm every year worldwide, more than 1 million die before the age of $5 \mathrm{y}$ due to preterm birth and associated complications. Although preterm survival rates have increased in high-income countries, they still die due to lack of adequate care in many low- and middle-income countries [1]. India accounts for $33 \%$ of the total global deaths due to preterm births [2]. Appropriate training at the grass-root level and availability of basic infrastructure at all delivery points will improve the survival of this vulnerable cohort. Providing optimal care of preterm neonates is a joint responsibility of all stakeholders including doctors, nurses, and mothers. In this context, we appreciate the innovative interactive e-learning method developed by National Neonatology Forum Network to disseminate knowledge to a larger group of participants over a wide geographical area [3].

The resource material used for this course was a standardized educational package on "Facility-based Care of Preterm Infant," which was hosted on Telegram, a cloud-based instant messaging platform. This learning module utilized participatory learning methodology including self-directed reading, poster demonstration, 110 videos, 55 webinars, and self-evaluation by multiple choice questions. A Telegram group "NNF Preterm Care E Module" was created by the central coordinating team, and facilitators were added. Subsequently, the participants were enrolled in the group by the facilitators and included in-service nurses from India, Bhutan, Bangladesh, and UAE. The 10 modules spread over $10 \mathrm{wk}$, were uploaded by central coordinating team. The resource material each week consisted of webinars, videos, and other reading materials. Mentors specified for that week facilitated learning through discussions. There was a live session every week to address any additional queries and to summarize the key messages of the week. Quiz bot, a

Adhisivam Bethou

adhisivam1975@yahoo.co.uk

1 Department of Neonatology, Jawaharlal Institute of Postgraduate Medical Education and Research (JIPMER), Pondicherry 605006, India third-party tool on Telegram, was used to create timed multiple choice question assessments. The authors evaluated the chats, discussion posts, and quiz attempt each week as a surrogate marker for ongoing attention and interest of the participants in the course. A designated central coordinating team monitored enrollment, tracked participant responses, and co-ordinated overall assessment. Among the 4623 nursing professionals who participated, majority felt that the topics were relevant and rated their overall experience as very good to excellent [3].

Enhancing the knowledge of professionals in supporting preterm neonates is of growing significance internationally. The sustained increase in preterm birth rates coupled with the ongoing improvement in survival rates for extremely preterm babies implies that there are increasing numbers of preterm survivors entering societies every year [4]. It is really amazing that technology including web-based e-learning can be utilized to train health care workers for practicing standard protocols within a reasonably short time frame. It is definitely cost-effective considering the output and large number of beneficiaries. Especially, during unusual situations like the COVID pandemic, e-learning is indispensible. Challenges do exist with all interventions and training techniques. How the knowledge gained through this novel mobile phone-based interactive e-learning platform is transformed to practical preterm care in the field should also be evaluated.

\section{Declarations}

Conflict of Interest None.

\section{References}

1. Chawanpaiboon S, Vogel JP, Moller AB, et al. Global, regional, and national estimates of levels of preterm birth in 2014: a systematic review and modelling analysis. Lancet Glob Health. 2019;7:e37-46. 
2. Walani SR. Global burden of preterm birth. Int J Gynaecol Obstet. 2020;150:31-3.

3. Anand P, Thukral A, Deorari AK; National Neonatology Forum Network. Dissemination of best practices in preterm care through a novel mobile phone-based interactive e-learning platform. Indian J Pediatr. 2021. https://doi.org/10.1007/s12098-021-03689-6.

4. Johnson S, Bamber D, Bountziouka V, et al. Improving developmental and educational support for children born preterm: evaluation of an e-learning resource for education professionals. BMJ Open. 2019;9:e029720.

Publisher's Note Springer Nature remains neutral with regard to jurisdictional claims in published maps and institutional affiliations. 